\title{
The Major Leukocyte Chemotactic and Activating Factors in the Mouse Gut Lumen Are Not N-Formylpeptide Receptor 1 Agonists
}

\author{
Teresa Ojode Erich H. Schneider H. Lee Tiffany Sunny Yung Ji-Liang Gao \\ Philip M. Murphy
}

Molecular Signaling Section, Laboratory of Molecular Immunology, National Institute of Allergy and Infectious Diseases, National Institutes of Health, Bethesda, Md., USA

\section{Key Words}

Microbiome $\cdot$ Chemotaxis $\cdot$ Inflammation $\cdot$ Formylpeptide $\cdot$ G-protein-coupled receptor

\begin{abstract}
Cultured bacteria release $\mathrm{N}$-formylpeptides, which are potent chemoattractants for phagocytic leukocytes acting at G-protein-coupled receptors FPR1 and FPR2. However, the distribution and immunologic activity of these molecules at mucosal surfaces, where large numbers of bacteria are separated from the immune system by epithelium, remain undefined. To investigate this for the gut, we tested leukocyte responses to cell-free gut luminal contents from C57BI/6 mice fed a chow diet. Small and large intestine contents were able to compete with labeled $\mathrm{N}$-formylpeptide for binding to FPR1, indicating the presence of FPR1 ligands in the gut lumen. Material from both small and large intestine induced robust calcium flux responses by primary FPR $1^{+}$leukocytes (mouse bone marrow cells and splenocytes and human peripheral blood neutrophils and mononuclear cells), as well as chemotactic responses by both mouse bone marrow cells and human peripheral blood neutrophils. However, unlike defined $\mathrm{N}$-formylpeptides, calcium flux responses induced by gut luminal contents were insensitive both to pertussis toxin treatment of leukocytes and to proteinase $\mathrm{K}$ digestion
\end{abstract}

of the samples. Moreover, the gut samples were fully active on neutrophils from mice lacking Fprl, and the kinetics of the calcium flux response differed markedly for neutrophils and peripheral blood mononuclear cells. The active factor(s) could be dialyzed using a 3.5-kDa pore size membrane. Thus, mouse intestinal lumen contains small, potent and highly efficacious leukocyte chemotactic and activating factors that may be distinct from neutrophils and peripheral blood mononuclear cells and distinct from Fpr1 agonists.

Copyright $\odot 2012$ S. Karger AG, Basel

\section{Introduction}

In the normal gastrointestinal tract, the lumen contains commensal microorganisms (the microbiome), food, mucus, exocrine secretions and waste products, as well as factors released by both microbes and gastrointestinal tissue. The microbiome in particular consists of a highly diverse community of bacteria, which together exceed the total number of human cells by more than 10 -fold [1]. The microbiome has a number of important salutary functions, for example in nutrition, to produce vitamins and to facilitate digestion of complex plant polysaccharides, as well as in host defense, to facilitate development and function of the mucosal immune system [1-3].

\section{KARGER}

Fax +41613061234

E-Mail karger@karger.ch

www.karger.com
(C) 2012 S. Karger AG, Basel

$1662-811 \mathrm{X} / 13 / 0051-0002 \$ 38.00 / 0$

Accessible online at:

www.karger.com/jin
Dr. Philip M. Murphy

Molecular Signaling Section, Laboratory of Molecular Immunology

National Institute of Allergy and Infectious Diseases, National Institutes of Health

Bldg. 10, Room 11N113, Bethesda, MD 20892 (USA)

Tel. +1 301496 8616, E-Mail pmm@nih.gov 
The molecular and cellular mechanisms by which the microbiome modulates mucosal immunity have been the subject of intense research, but are still poorly understood. Bacteria release multiple constituents, known collectively as pathogen-associated molecular patterns (PAMPs), which stimulate specific pattern recognition receptors on leukocytes and other cell types. PAMPs include polynucleotides, peptidoglycan, lipopolysaccharide (LPS), flagellin, muramyl dipeptides, and pathogenspecific oligonucleotides such as hypomethylated $(\mathrm{CpG})$ DNA, all of which activate specific Toll-like receptors [4, 5]. In addition, bacterial PAMPs may include $\mathrm{N}$-formylpeptides, a subset of bacterial protein breakdown products that activate pertussis toxin-sensitive G-proteincoupled phagocyte chemotactic receptors named 'N-formylpeptide receptors' (FPRs) [6, 7]. Two principal FPRs have been identified in humans, FPR1 and FPR2/ALX (Fpr1 and Fpr2 in mouse) [7]. The normally harmonious coexistence of the microbiome in the gut lumen with leukocytes in gut tissue may be accounted for in part by an intact epithelium; however, when this breaks down, for example in inflammatory bowel disease, unrestricted communication between the two sets of cells may occur [8]. In the present study, we hypothesized that gut bacteria release $\mathrm{N}$-formylpeptides in situ that are able to activate leukocytes bearing FPRs. We show instead that while the gut lumen does in fact contain Fprl ligands and potent leukocyte chemotactic and activating factors, surprisingly the activity appears to be independent of Fprl.

\section{Materials and Methods}

\section{Agonists}

N-formyl-Met-Leu-Phe (fMLF), interleukin (IL)-8 and macrophage inflammatory protein 2 (MIP-2) were obtained from $\mathrm{Pe}$ protech (Rocky Hill, N.J., USA). fNLFNYK-fl and ATP were obtained from Invitrogen (Carlsbad, Calif., USA). Leukotriene $\mathrm{B}_{4}$ $\left(\mathrm{LTB}_{4}\right)$ was obtained from Cayman Chemical Company (Ann Arbor, Mich., USA).

\section{Mouse Gut Lumen Content}

Luminal contents from small and large intestine were harvested from wild-type C57BL/6NTac mice from Taconic Farms (Hudson, N.Y., USA) or from $\mathrm{Fprl}^{-/-}$mice backcrossed 10 generations onto the C57BL/6NTac background. The construction of Fpri-1mice has been described previously [9]. Both strains of mice were produced at Taconic Farms and transferred to the National Institute of Allergy and Infectious Diseases for experiments, which were approved by the National Institute of Allergy and Infectious Diseases Animal Care and Use Committee. Small intestine content (SC) was defined as luminal contents from the gastroduodenal junction to the ileocecal junction. Large intestine content (LC) was defined as luminal contents from the ileocecal junction to the rectoanal junction. Each segment was flushed four times with $4 \mathrm{ml} 1 \times$ PBS using a shielded IV catheter (BD Insyte ${ }^{\mathrm{TM}}$ Autoguard $^{\mathrm{TM}}$, Franklin Lakes, N.J., USA); the washings were mixed and centrifuged at $12,000-15,000 \mathrm{rpm}$ for $10 \mathrm{~min}$ twice to separate soluble factors from particulates. Supernatants from processed SC and LC were stored at $-20^{\circ} \mathrm{C}$ until use. To estimate the molecular size of leukocyte-activating factors in the mouse gut lumen, we dialyzed $0.5 \mathrm{ml}$ of SC or LC in dialysis cassettes (Pierce, Rockford, Ill., USA) immersed in 1 liter PBS stirred for two consecutive 2-hour periods at $25^{\circ} \mathrm{C}$, followed by one overnight period at $4^{\circ} \mathrm{C}$; the buffer was refreshed for each period. For some experiments, mouse gut lumen extracts and control chemoattractants were incubated with $800 \mu \mathrm{g} / \mathrm{ml}$ proteinase K (Cat. No. P8102S from New England Biolabs, Ipswich, Mass., USA) for $>2 \mathrm{~h}$ at $55^{\circ} \mathrm{C}$. Digested proteins were placed in loading buffer and resolved by SDS-PAGE; gels (Cat. No. 161-1106 from Bio-Rad Laboratories, Hercules, Calif., USA) were stained with Coomassie blue. Digested proteins were also used directly as test stimuli on cells in the calcium flux assay, as described below.

\section{Cells}

Primary leukocytes were obtained from bone marrow and spleen either from wild-type C57BL/6NTac mice or from $\mathrm{Fprl}^{-/-}$ mice backcrossed on the C57BL/6NTac background. Bone marrow cells were obtained by flushing the hind-limb femur and tibia with PBS. Spleens were mechanically disrupted in PBS. To obtain single-cell suspensions, bone marrow cells and splenocytes were passed through a $100-\mu \mathrm{m}$ nylon cell strainer. Residual erythrocytes were removed by $0.2 \%$ hypotonic $\mathrm{NaCl}$ lysis. Cells were pelleted, then washed in PBS and passed through a $40-\mu \mathrm{m}$ nylon cell strainer to remove debris. Human leukocytes were harvested from $15 \mathrm{ml}$ of heparinized whole blood from anonymous healthy donors recruited by the Department of Transfusion Medicine at the Warren G. Magnuson Clinical Center, National Institutes of Health. Peripheral blood mononuclear cells (PBMCs) were separated from neutrophils and erythrocytes using lymphocyte separation medium (Lonza, Walkersville, Md., USA) according to the instructions of the manufacturer, then washed in PBS. Neutrophils were separated from erythrocytes by 3\% dextran sedimentation and hypotonic $\mathrm{NaCl}$ lysis, then washed in $\mathrm{PBS}$. Human embryonic kidney (HEK) 293 cells, obtained from ATCC, were stably transfected with a plasmid encoding human FPR1, as previously described [10]. Cells were cultured in Dulbecco's modified Eagle's medium (Invitrogen) $+15 \% \mathrm{FBS}+2 \mathrm{mg} / \mathrm{ml} \mathrm{G} 418$ at $37^{\circ} \mathrm{C}$ in an atmosphere of $100 \%$ humidity and $5 \% \mathrm{CO}_{2}$. The medium was changed every 3 days.

\section{Calcium Flux Assay}

Calcium flux was measured in cells using a FlexStation ${ }^{\circledR} 3$ microplate reader and the FLIPR Calcium 3 Assay Explorer Kit (Molecular Devices, Sunnyvale, Calif., USA). Cells were suspended in HBSS (with $\mathrm{Ca}^{2+}+\mathrm{Mg}^{2+}$ ) and incubated for at least $30 \mathrm{~min}$ with an equal volume of FLIPR Calcium Assay 3 Dye (component A). The final total volume of $200 \mu \mathrm{l}$ containing $2.5-10 \times 10^{5}$ cells per well was analyzed by FlexStation using an excitation wavelength of $485 \mathrm{~nm}$ and an emission wavelength of $525 \mathrm{~nm}$. Test substances were diluted in HBSS (with $\mathrm{Ca}^{2+}+\mathrm{Mg}^{2+}$ ) or PBS and added to the wells robotically. In some experiments, test substances were incubated in a boiling water bath for a minimum of $10 \mathrm{~min}$ prior to use. 
Chemotaxis Assay

Leukocytes (human neutrophils or mouse bone marrow cells) were washed in PBS and suspended in chemotaxis buffer [0.5\% bovine serum albumin (fraction V) + RPMI-1640 + $10 \mathrm{mM}$ HEPES]. Chemotaxis was measured using 6.5-mm Transwells ${ }^{\circledR}$ with either 5 - or $8-\mu \mathrm{m}$ pore polycarbonate membrane inserts (Corning, Lowell, Mass., USA). Test substances in $600 \mu \mathrm{l}$ chemotaxis buffer were added to the bottom of each chamber and 5-10 $\times 10^{5}$ cells suspended in $100 \mu \mathrm{l}$ of buffer were placed at the top. Cells were incubated at $37^{\circ} \mathrm{C}, 5 \% \mathrm{CO}_{2}$ for $3 \mathrm{~h}$. Samples from the bottom were collected and mixed with $10.2 \mu \mathrm{m}$ Sphero $^{\mathrm{TM}}$ AccuCount blank particles (Spherotech, Lake Forest, Ill., USA) and counted using a FACScalibur flow cytometer to determine the number of migrating cells. In some experiments, cells were preincubated in control buffer $(1 \times$ PBS) or $250 \mathrm{ng} / \mathrm{ml}$ pertussis toxin (Cat. No. 180 from List Biological Laboratories, Campbell, Calif., USA) in $\mathrm{PBS}$ at $37^{\circ} \mathrm{C}$ for $4 \mathrm{~h}$ prior to measuring chemotaxis. Data are presented as the chemotactic index.

\section{Binding Assay}

To determine whether FPR1 ligands are present in mouse gut lumen, we measured equilibrium binding of the fluorescently labeled FPR1 ligand fNLFNYK-fl to FPR1-transfected HEK 293 cells in the presence and absence of fMLF and/or SC and LC. Total binding was determined after addition of $2 \mathrm{nM}$ fNLFNYK- $f l$ to FPR1-bearing cells in the absence of FPR1 ligands. The $\mathrm{K}_{\mathrm{d}}$ value of $\mathrm{fNLFNYK-fl}$ at FPR1 expressed on HEK 293 cells is approximately $2.7 \mathrm{nM}$ (determined by saturation binding assays, data not shown). Therefore, an fNLFNYK-fl concentration of $2 \mathrm{nM}$ ensures high sensitivity of the assay. The positive control of $10 \mu \mathrm{M}$ fMLF fully displaced $2 \mathrm{nM}$ fNLFNYK- $f l$ and was used to define specific binding. To ensure the binding activity was FPR1 specific, we used untransfected HEK 293 cells as negative control. The binding assay was carried out in a total volume of $300 \mu$ l with $1.2 \times$ $10^{5}$ cells in a buffer composed of $0.1 \%$ bovine serum albumin $($ fraction $\mathrm{V})+1 \times$ HBSS $+20 \mathrm{mM}$ HEPES on ice in the dark for 15 min. SC and LC were added at the beginning of the incubation to achieve a final dilution of $1: 10$. Data are presented as the percent of total specific binding.

\section{Statistical Methods}

Results are shown as means \pm SEM. Differences between groups were compared using Student's t test (two sets of data) or one-way ANOVA (three or more sets of data), followed by the Student-Newman-Keuls post-hoc test. Differences were considered significant at $\mathrm{p}<0.05$.

\section{Results}

\section{The Lumen of the Mouse Gastrointestinal Tract}

\section{Contains FPR1 Ligands}

To determine whether FPR1 ligands are present in the lumen of the mouse gastrointestinal tract, we collected the contents of the small and large bowel separately, removed particulates by centrifugation and conducted competitive ligand binding studies with the supernatants

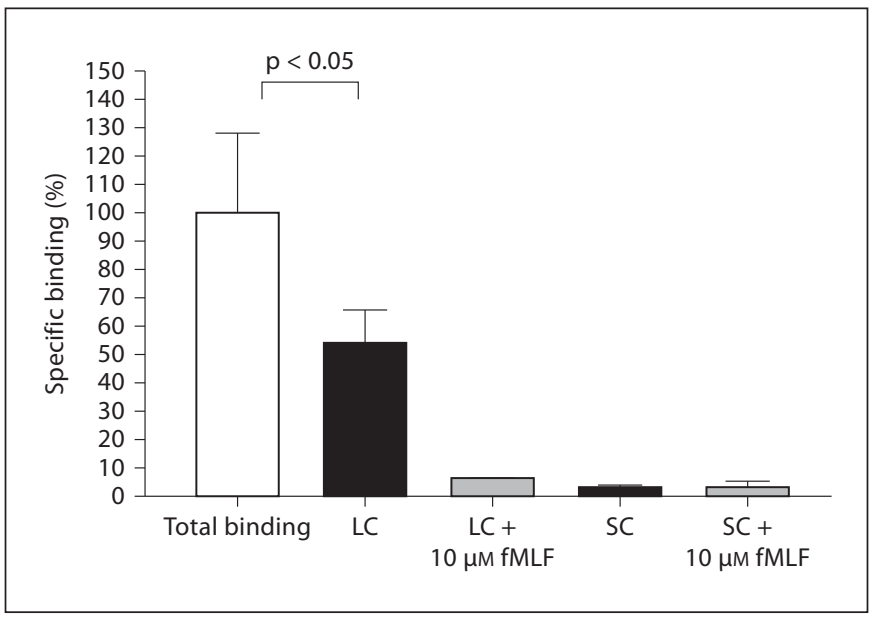

Fig. 1. The lumen of the mouse small and large bowel contains FPR1 ligands. FPR1 on transfected HEK 293 cells was labeled with fNLFNYK- $f l$ and binding was measured in the presence and absence of the indicated substances by FACS. Data are the mean \pm SD from 1 experiment, each condition tested in triplicate, and are representative of 4 independent experiments. SC and LC were tested at a final dilution of 1:10.

using the fluorescent formylpeptide fNLFNYK-fl to label human FPR1 on FPR1-transfected HEK 293 cells. We will refer to these supernatants hereafter as SC and LC. As expected, fNLFNYK- $f l$ specifically bound to FPR1-transfected cells. Binding was substantially reduced by SC, indicating the presence of FPR1 ligands, but surprisingly less so by LC, though still statistically significant (fig. 1). To test the possibility that formylpeptide scavengers or FPR1 antagonists potentially present in LC reduce probe binding, we conducted the binding assay with LC in the presence of $10 \mu \mathrm{M}$ fMLF. Under these conditions, fNLFNYK- $f$-specific binding was reduced to approximately $5 \%$ of control, suggesting that the failure of LC to fully reduce probe binding to FPR1 is most likely due to the absence of high-affinity FPR1 ligands in LC. fMLF had no observable effect on probe binding when mixed with SC, which was already about $95 \%$ reduced by SC alone. Thus, SC but not LC appears to contain high-affinity FPR1 ligands.

\section{The Lumen of the Mouse Gastrointestinal Tract Contains Factors That Activate Mouse and Human Leukocytes}

To determine whether mouse gut luminal contents are able to activate leukocytes, we first measured calcium flux, which is induced in leukocytes by most chemoattractants $[7,10,11]$. Both SC and LC induced robust cal- 


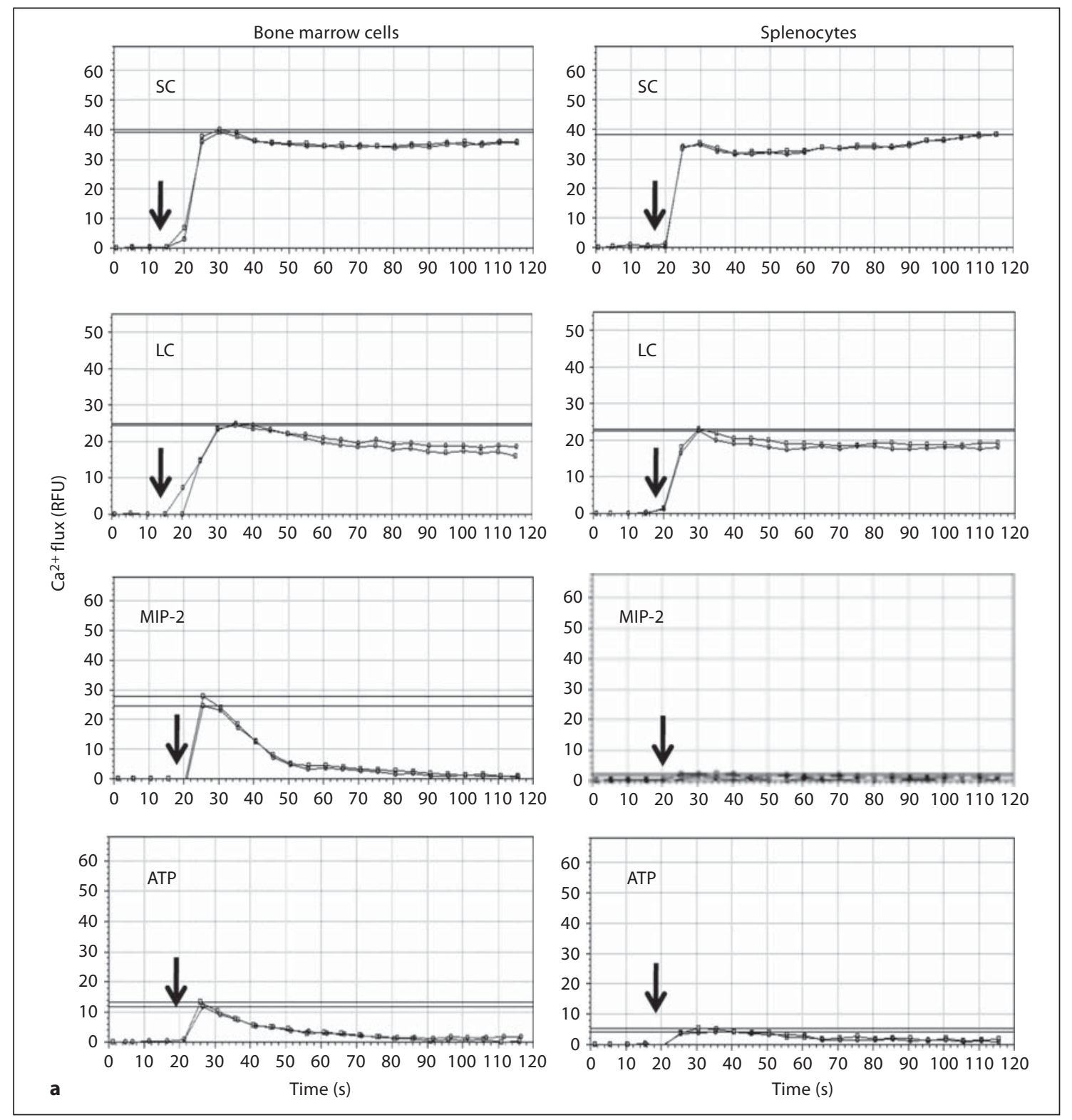

Fig. 2. The lumen of the mouse small and large bowel contains mouse leukocyte-activating factors. Calcium flux was measured in real time using the cells indicated at the top of each column as targets. SC and LC were tested at a final dilution of 1:10. Data are from a single experiment. RFU = Relative fluorescence units. a Kinetics: cells were stimulated at the times indicated by the arrows with the substances indicated at the upper left of each panel. Duplicate tracings are shown from the same experiment, which is representative of 5 and 12 independent experiments for splenocytes and bone marrow cells, respectively. Horizontal lines demarcate the peak response for each tracing.

cium flux responses in total mouse bone marrow cells and splenocytes (fig. 2), as well as in human peripheral blood neutrophils and PBMCs (fig. 3). SC and LC from all mouse donors tested $(n=25)$ were active on all leukocyte populations tested (fig. 2b, 3b). As expected, the efficacy of both SC and LC from different animals varied for all four leukocyte populations tested. With regard to the kinetics of the response, for mouse bone marrow cells and splenocytes as well as for human PBMCs, the increase in intracellular $\mathrm{Ca}^{2+}$ concentration induced by both $\mathrm{SC}$ and 
Fig. 2. The lumen of the mouse small and large bowel contains mouse leukocyte-activating factors. Calcium flux was measured in real time using the cells indicated at the top of each column as targets. SC and LC were tested at a final dilution of 1:10. Data are from a single experiment. $\mathrm{RFU}=$ Relative fluorescence units. b Efficacy: the peak calcium flux response for all mouse donors tested is summarized by the scatter plot. Horizontal lines indicate the peak calcium flux response for the positive and negative controls, as coded in the insets.

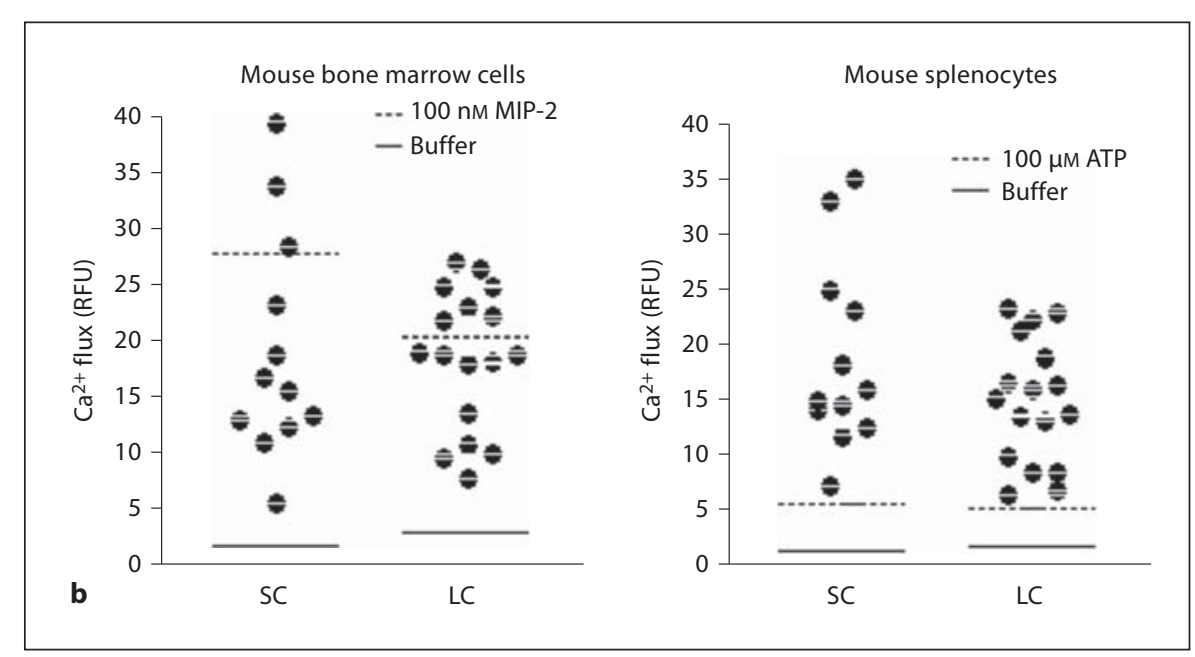

LC was both rapid and sustained (fig. 2, 3). This is atypical for leukocyte chemoattractants [7, 10, 11], as illustrated for the activation of mouse bone marrow cells by the neutrophil-targeted chemokine MIP-2, where the response is rapid but transient (fig. 2). SC also induced a rapid, sustained response in human neutrophils. In contrast, LC induced calcium transients in human neutrophils quite similar to the kinetics of those elicited by fMLF (fig. 3a) and chemokines such as IL-8 (data not shown).

For technical reasons, the highest concentration of SC and LC tested was a 1:10 dilution of the harvested and processed material, and this was also the dilution that resulted in the maximal calcium flux response. The range of peak responses of mouse bone marrow cells was similar for SC and LC and included the peak response induced by stimulation with a saturating concentration of $100 \mathrm{nM}$ MIP-2 (fig. 2b). The peak response range of mouse splenocytes was also similar for SC and LC and exceeded the maximal response to $100 \mu \mathrm{M} \mathrm{ATP}$, tested as a positive control (fig. 2b). For human PBMCs, the maximal response elicited by SC was much higher than that elicited by LC (fig. 3a), whereas for human neutrophils, SC and LC induced similar maximal responses that were similar to that induced by a saturating concentration of $10 \mathrm{nM}$ fMLF, tested as a positive control (fig. 3a). These data indicate that SC and LC both clearly contain leukocyteactivating factors; however, they behave kinetically in a manner that can be distinguished from classic chemoattractants and chemokines. In addition, they can be distinguished from each other by the efficacy observed across the four leukocyte populations tested, suggesting the presence of distinct factors in the small and large intestinal lumen.

To further examine the relationship of $\mathrm{N}$-formylpeptides to the calcium flux activity present in SC and LC, we performed extensive reciprocal desensitization experiments with human neutrophils as targets. We found that $0.1 \times$ of either SC or LC added first could reduce the calcium flux signal elicited by $1 \mathrm{nM}$ fMLF added second by $>75 \%$ relative to the control signal, but neither had any effect on the signal induced by $100 \mathrm{nM}$ fMLF added second; reciprocally, $50 \mathrm{nM}$ fMLF added first could reduce the calcium flux signal elicited by $0.1 \times$ of either SC or LC added second by $>75 \%$ compared to the control signal. This suggests pathways by which formylpeptide signaling may cross-regulate neutrophil agonists present in SC and LC. The data are consistent with FPRs as the relevant targets, but heterologous and cross-desensitization involving other receptors is also possible.

In chemotaxis assays, both SC and LC induced migration of mouse bone marrow cells (fig. 4); the threshold for LC activity fell between 0.001 and $0.01 \times$ dilution, similar to the calcium flux assay (data not shown). Since FPR1 ligands were found in SC (fig. 1), it was reasonable to hypothesize that the active factors might include but not be solely explained by classic $\mathrm{N}$-formylpeptides, and we addressed this question in the next series of experiments.

\section{Leukocyte-Activating Factors in the Gut Lumen Can}

Be Distinguished from Prototypic N-Formylpeptides

To assess the physical nature of leukocyte-activating factors in SC and LC, we first boiled the samples and assessed calcium-mobilizing activity using mouse bone 


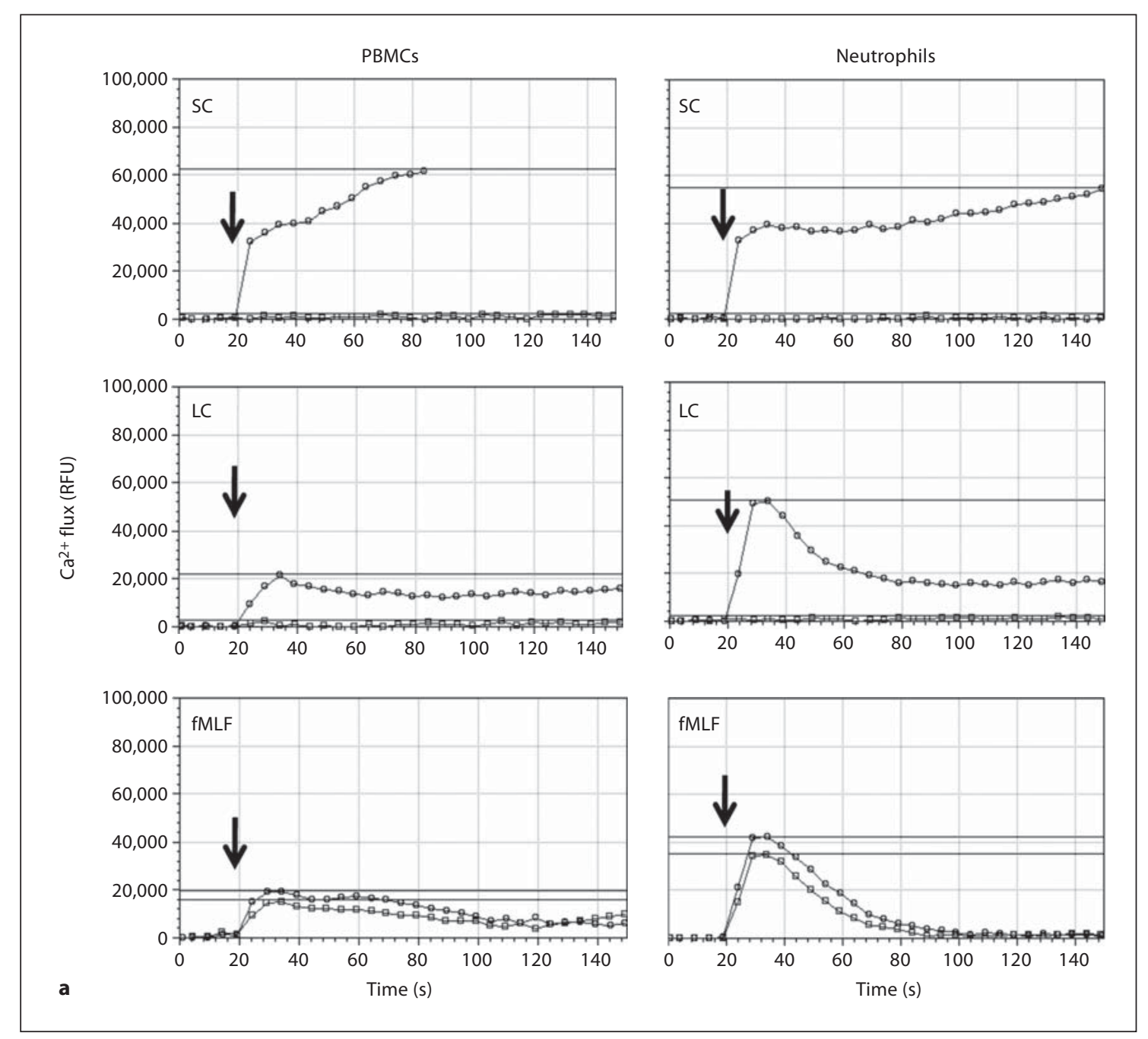

Fig. 3. The lumen of mouse small and large bowel contains human leukocyte-activating factors. Calcium flux was measured in real time using the cells indicated at the top of each column as targets. RFU $=$ Relative fluorescence units. a Kinetics: cells were stimulated at the times indicated by the arrows with the substances indicated at the upper left of each panel. SC and LC were from the same mouse and tested at a 1:10 dilution; fMLF was tested at 50
nM. Tracings shown are from the same experiment, and are representative of 5 and 6 independent experiments for PBMCs and polymorphonuclear neutrophils, respectively. Horizontal lines demarcate the peak response for each tracing. For SC and LC, open circles denote the test substance indicated in the upper left of each panel, and open squares denote the unstimulated control. For fMLF, the data in each panel denote duplicate tests of fMLF. marrow cells as targets. In control studies, we found, as expected, that boiling markedly diminished the activity of MIP-2, which is a large folded protein chemoattractant (fig. 5), while having little effect on the activity of the small prototypic $\mathrm{N}$-formylpeptide chemoattractant $\mathrm{fMLF}$ or the lipid $\mathrm{LTB}_{4}$. Prolonged boiling also did not diminish the calcium-mobilizing activity of either SC or LC, suggesting that the activity could be due to $\mathrm{N}$-formylpeptides, but that it is unlikely to be due to a large protein, such as a chemokine. In fact, the activity was increased by boiling, suggesting the possibility that an inhibitory factor had been removed. To estimate the size of the leukocyte-activating factor(s) in LC, we dialyzed the samples using dialysis cassettes with 2-, 3.5- and 7-kDa pore cutoffs. We found that the great majority of the calcium flux activity for human neutrophils was lost when samples were dialyzed with $\geq 3.5-\mathrm{kDa}$ pore cutoffs (fig. 6). About half the activity was lost when the samples were 


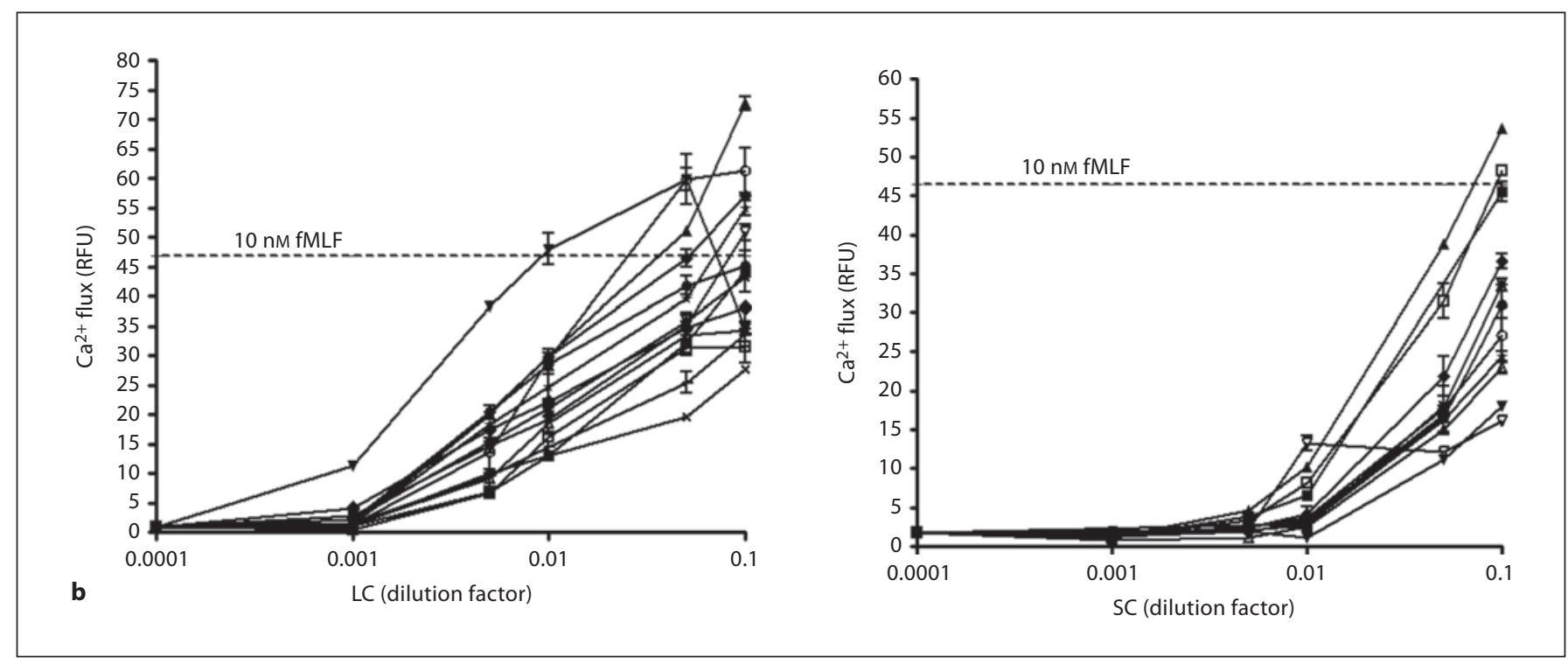

Fig. 3. The lumen of mouse small and large bowel contains human leukocyte-activating factors. Calcium flux was measured in real time. RFU = Relative fluorescence units. $\mathbf{b}$ Potency and efficacy: the peak response of human polymorphonuclear neutrophils is plotted as a function of serial dilutions of SC or LC used as stimuli for all mouse donors tested. Each line represents data from a single mouse. Horizontal lines indicate the peak response to 10 nM fMLF, tested as the positive control. dialyzed using a $2-\mathrm{kD}$ a pore cutoff. The same pattern was obtained when SC was dialyzed and tested for calciummobilizing activity with human neutrophils (data not shown).

To investigate directly whether the leukocyte-activating factors in SC and LC actually included agonists for FPRs, we compared SC- and LC-induced calcium flux responses in bone marrow cells from wild-type and $\mathrm{Fprl}^{-1-}$ mice. As expected, we observed a complete loss of responsiveness to fMLF in the knockout cells. In contrast, no significant differences in responsiveness were observed for wild-type versus knockout cells when either SC or LC were tested (fig. 7), suggesting that the active factors were not predominantly Fprl agonists. We were unable to determine whether LC or SC contained functional FPR agonists using a gain-of-function approach since SC and LC both induced calcium flux equally well in untransfected and FPR1- and FPR2-transfected HEK 293 cells, presumably by acting at a native receptor (data not shown).

$\mathrm{N}$-formylpeptides, other classical chemoattractants and chemokines all signal through Gi-type $\mathrm{G}$ proteins in a classic pertussis toxin-sensitive pathway [7]. However, surprisingly, we found that pertussis toxin treatment had no significant inhibitory effect on either SC- or LC-in-

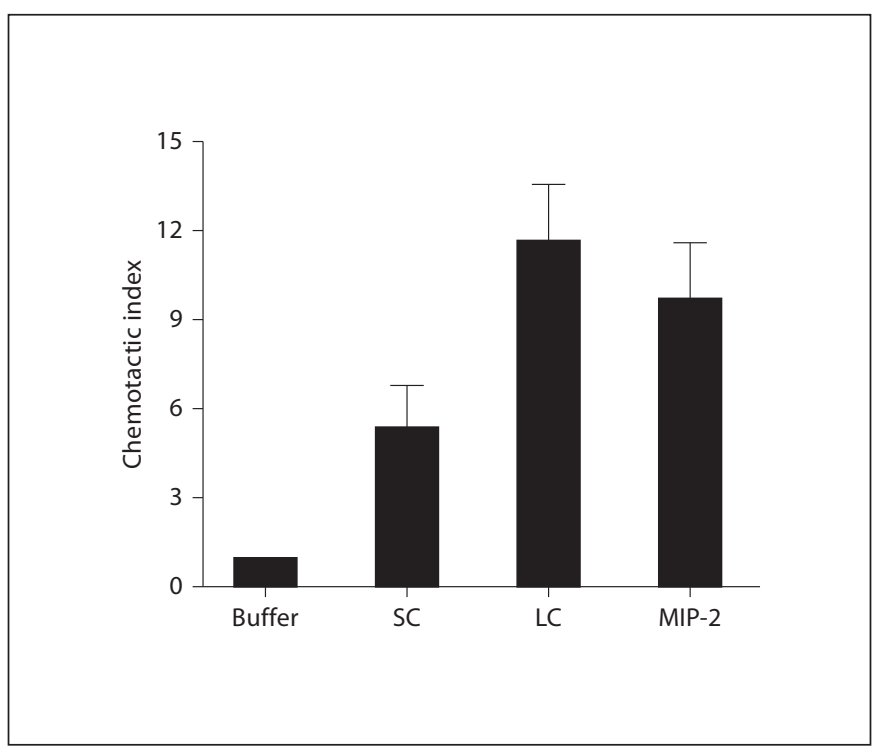

Fig. 4. The lumen of mouse small and large bowel contains chemotactic factors for mouse bone marrow cells. SC and LC were added to a final dilution of 1:10. MIP-2 was tested at $100 \mathrm{nM}$. Data are the mean $\pm \mathrm{SD}$ of duplicate determinations for each condition and are representative of 7 independent experiments. 


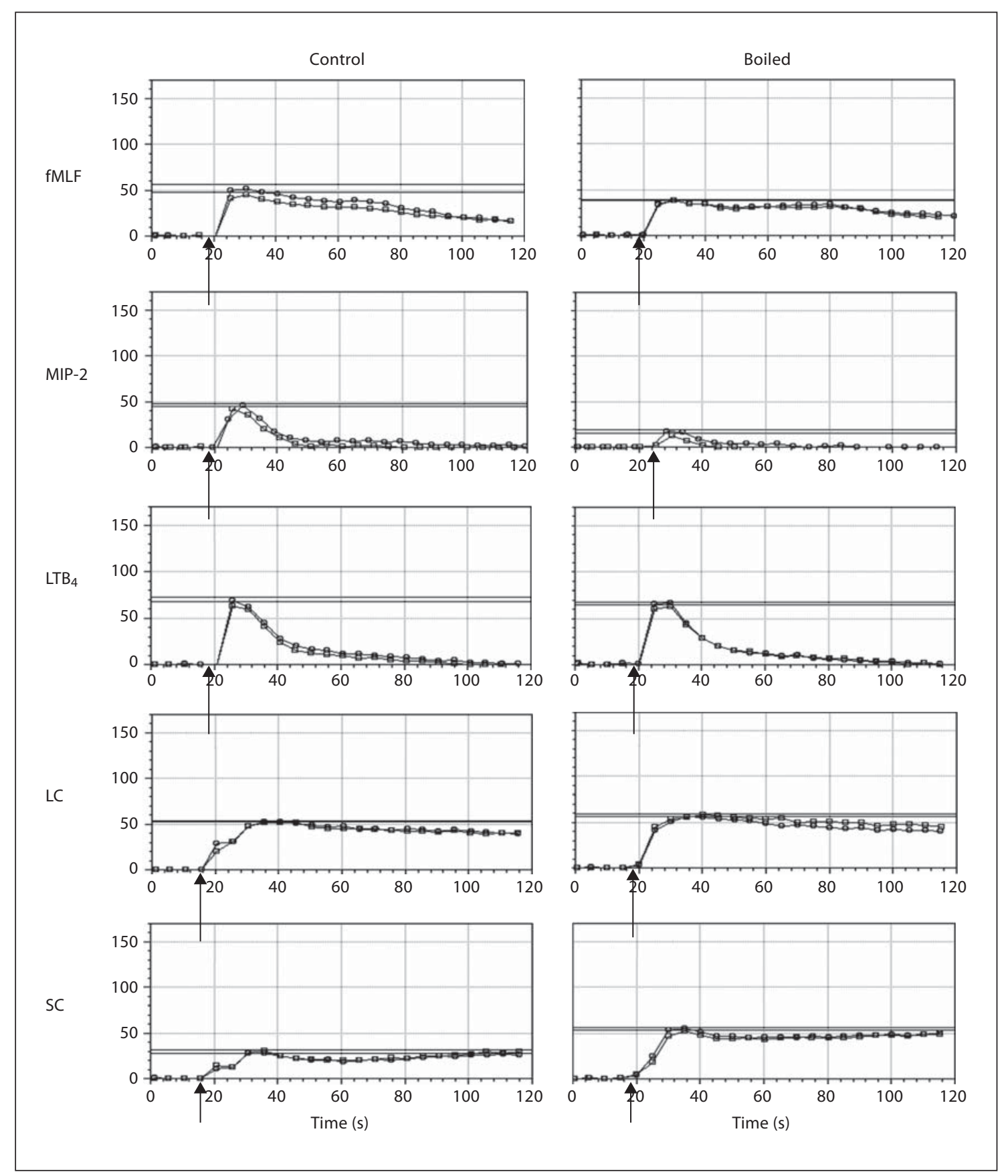

Fig. 5. Leukocyte-activating factors from the lumen of the mouse gastrointestinal tract are temperature resistant. Calcium flux was measured in real time using mouse bone marrow cells as targets. SC and LC were tested at a 1:10 dilution, $\mathrm{LTB}_{4}$ at $30 \mathrm{nM}, \mathrm{fMLF}$ at $10 \mu \mathrm{M}$, and MIP-2 at $50 \mathrm{nM}$. Kinetics: cells were stimulated at the times indicated by the arrows with the substances indicated at the left of each row. Before addition to the cells, each substance was either boiled for a minimum of $10 \mathrm{~min}$ or not boiled (control) as indicated in the column heading. Data are duplicate tracings all from the same experiment, which is representative of 9 independent experiments. Horizontal lines demarcate the peak response for each tracing. 


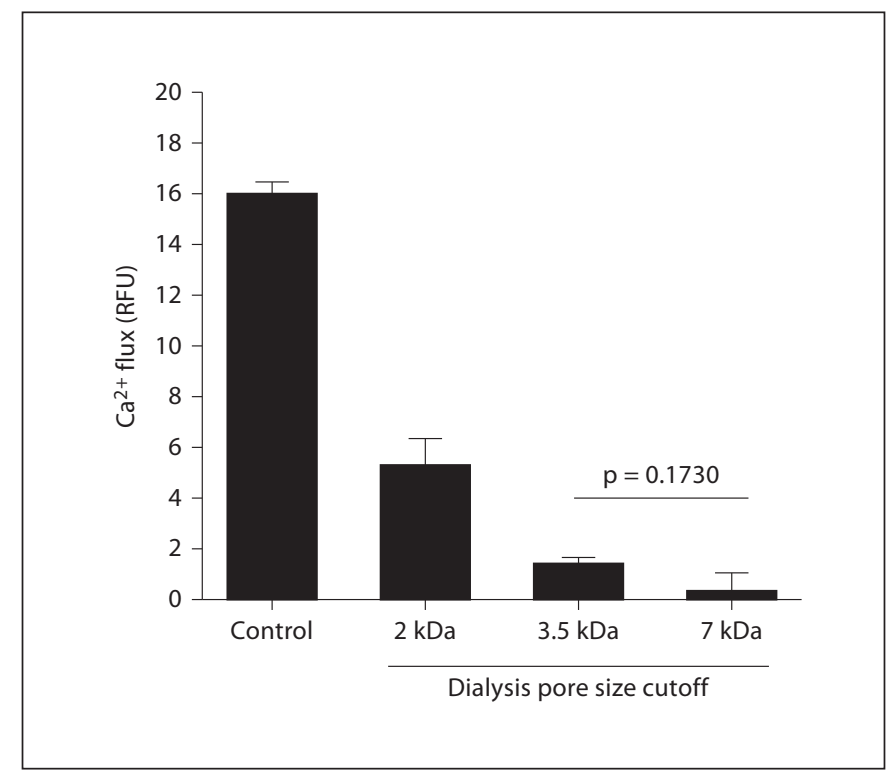

Fig. 6. Leukocyte-activating factors from the lumen of the mouse gastrointestinal tract are small dialyzable factors. Calcium flux was measured using mouse bone marrow cells as targets; LC was tested at a 1:10 dilution. Peak calcium flux response is plotted as the mean \pm SD from 1 experiment representative of 3 experiments. 'Control' denotes undialyzed LC. RFU = Relative fluorescence units.

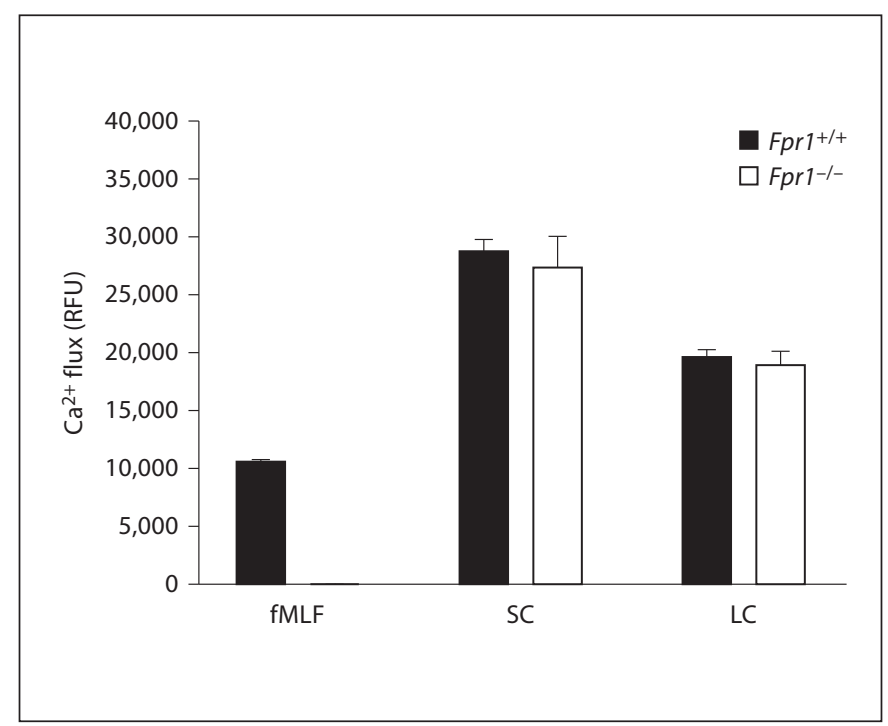

Fig. 7. Leukocyte-activating factors from the lumen of the mouse gastrointestinal tract are not solely Fprl agonists. Calcium flux was measured using mouse bone marrow cells from wild-type and $\mathrm{Fpr}^{-1-}$ mice as targets; LC and SC were tested at a 1:10 dilution, fMLF at $1 \mu \mathrm{M}$. Peak responses are plotted for duplicate determinations of each condition from 1 experiment. RFU = Relative fluorescence units. duced chemotaxis of mouse bone marrow cells (fig. 8). In contrast, in control experiments, pertussis toxin completely blocked fMLF-induced chemotaxis of bone marrow cells from both wild-type and $\mathrm{FprI}^{-/-}$mice across the entire concentration range tested, $10 \mathrm{nM}$ to $100 \mu \mathrm{M}$, as expected. As previously reported, the threshold for fMLF responsiveness of neutrophils from $\mathrm{Fprl}^{-/-}$mice shifted from 1 to $100 \mu \mathrm{M}$ relative to responses of cells from wildtype mice. This is most likely because fMLF responsiveness in $\mathrm{Fprl}^{-1-}$ mice is mediated by expression of Fpr2, which has a lower affinity than Fprl for fMLF [12].

To test whether the leukocyte-activating factors in SC and LC are proteins, we digested the samples with 800 $\mu \mathrm{g} / \mathrm{ml}$ proteinase $\mathrm{K}$. Coomassie blue-stained gels showed that SC and LC contained a complex mixture of proteins that were no longer detectable in samples treated with proteinase K (fig. 9a). We tested the biological activity of the samples by calcium flux assay of human peripheral blood neutrophils. Proteinase K treatment completely abolished fMLF- and IL-8-induced calcium flux in human neutrophils, but had no significant effect on either SC- or LC-induced calcium flux (fig. 9b, c). Recent reports have identified short-chain fatty acids produced by intestinal bacteria that are potent activators of calcium flux in neutrophils [13]. However, we found that one such short-chain fatty acid, acetate, was unable to desensitize neutrophil responses to SC (data not shown). Moreover, acetate, unlike SC and LC, activates neutrophils in a pertussis toxin-sensitive manner, suggesting that the activity in SC is unlikely to be due to acetate. We also tested ATP as a candidate, but in sequential stimulation experiments it had little to no effect on mouse bone marrow cell calcium flux responses to either SC or LC (data not shown).

\section{Discussion}

In the present study, we have shown that the mouse gut lumen contains potent chemotactic and activating factors for multiple leukocyte subsets in mouse and human, but that little if any of the activity may be attributed to protein agonists of Fprl. This result is surprising since (1) the gut lumen contains a massive number of bacteria [1], (2) bacteria initiate protein synthesis with $\mathrm{N}$-formylmethionine [6], (3) cultured bacteria generate and release N-formylpeptides [14], (4) FPR1 ligands are present in the gut lumen (fig. 1), (5) phagocytic leukocytes are sensitive to a wide range of tested $\mathrm{N}$-formylpeptides acting at Fprl [7, $11,15]$, and (6) intestinal epithelial cells express functional Fpr1 [16]. 


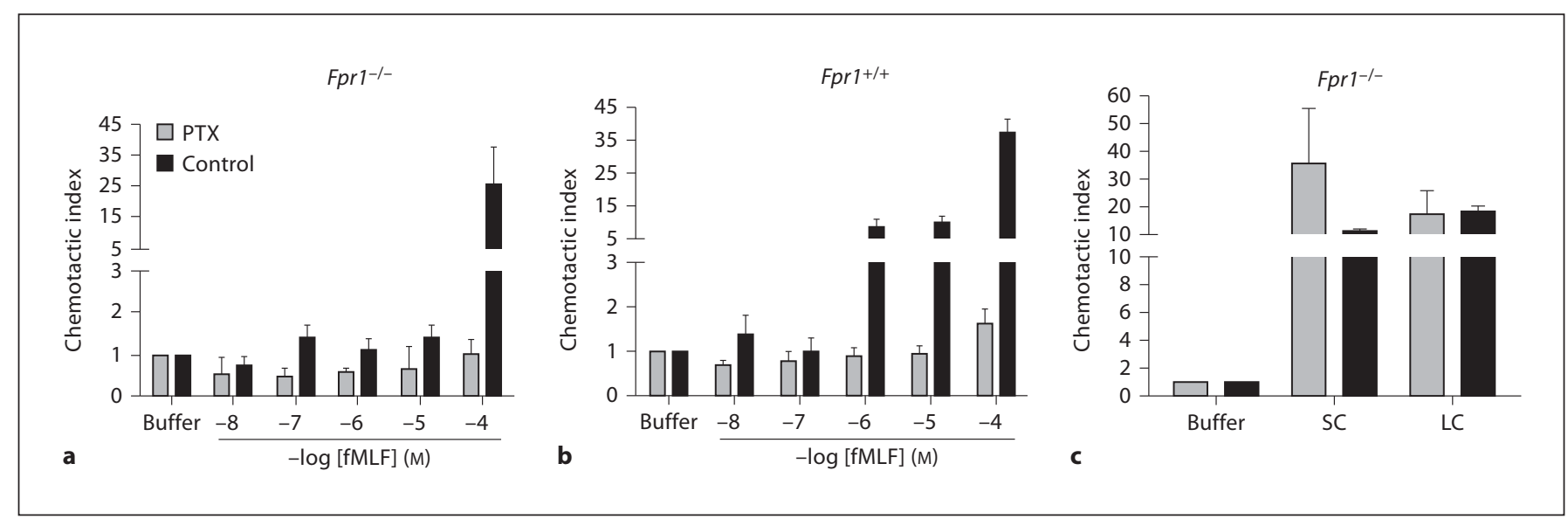

Fig. 8. Leukocyte chemotactic responses to luminal contents of the mouse gastrointestinal tract are pertussis toxin (PTX) resistant. Chemotaxis was measured using bone marrow cells from wild-type $\left(\mathrm{Fprl}^{+/+}\right)$and $\mathrm{Fprl}^{-/-}$ mice as targets. Cells were tested after PTX pretreatment (shaded bars) or control buffer pretreatment (black bars). fMLF was tested at the indicated concentrations (a, b); LC and SC were tested at a 1:10 dilution (c). Data are the mean \pm SD from 1 experiment representative of 2 independent experiments.

Our findings extend previous work by others demonstrating that bacteria are able to directly produce or indirectly induce production of leukocyte-activating factors $[6,13,17]$. Most previous studies have approached this question by isolating specific microorganisms from the gut and other sites for the study of secreted factors, whereas we have used an unbiased approach to study leukocyteactivating factors produced and released in situ throughout the gut lumen. Since we have not yet specifically identified the active factors at the molecular level, we do not yet know the number of factors, their relative activity in the mix, or their exact provenance. Nevertheless, our finding of differential efficacy and kinetics in the calcium flux assay for mouse bone marrow cells and splenocytes and human PBMCs and neutrophils suggests that there may be multiple active factors in the lumen able to act not only on gut epithelial cells (data not shown), but also on multiple subtypes of leukocytes that may patrol the gut normally and accumulate in it during disease. Several leukocyte-activating factors have already been defined in the gut, including hepoxilin A3 and short-chain fatty acids, which are produced by the gut mucosa and flora, respectively; however, their relative contribution to the activity we have described has not been defined $[13,18]$. Superantigens can also induce calcium flux but are large protease-sensitive proteins and would not be expected to account for the chemotactic activity we describe. The inability of the short-chain fatty acid acetate to affect sig- naling induced by gut luminal content (data not shown) or to chemoattractant leukocytes (data not shown) suggests that it also does not solely account for SC or LC activity. LPS was also inactive (data not shown). Additional sources that must be considered include food metabolites, waste products released into the gut and exocrine secretions.

Defining leukocyte-activating factors in the gut lumen may help explain the strong influence of commensal microorganisms on both mucosal immune system development and inflammatory bowel disease and is therefore an important goal in mucosal immunology $[8,19,20]$. The gut mucosa is separated from the lumen by a single layer of epithelial cells that are capable of sensing pathogen-specific molecules directly as well as by crosstalk with dendritic cells [21]. Overlaying the epithelial cell layer is a mucus coating secreted by goblet cells that contains antimicrobial peptides secreted by specialized gut epithelial cells, that together function as a protective barrier reducing direct bacterial contact with the epithelial cell layer [22]. Interspersed within the epithelial cell layer are intraepithelial lymphocytes and dendritic cells.

In the normal, healthy gastrointestinal tract, the mucosal immune system is organized into discrete inductive sites, such as gut-associated lymphoid tissue, located throughout the gut, and Peyer's patches, found only in the distal ileum, as well as effector sites, which are distributed more diffusely throughout the mucosa. All of these 
Fig. 9. Leukocyte-activating factors from the lumen of the mouse gastrointestinal tract are proteinase $\mathrm{K}$ resistant. a Proteinase K digests SC and LC proteins. The substrates indicated at the bottom of the gel were incubated with proteinase $\mathrm{K}(+)$ or control buffer $(-)$. Digests were then analyzed by SDS-PAGE. M = Markers. b Neutrophil-activating factors in SC and LC are proteinase $\mathrm{K}$ resistant. Human neutrophils were stimulated with the substances indicated to the right of each row that had been incubated either with proteinase $\mathrm{K}$ or control buffer, as indicated at the top of each column of tracings, and calcium flux was measured. LC and SC were added at a final dilution of 1:10, and the undigested concentrations of fMLF and IL- 8 were both 100 $\mathrm{nM}$. Data are from 1 experiment that is representative of 4 independent experiments.

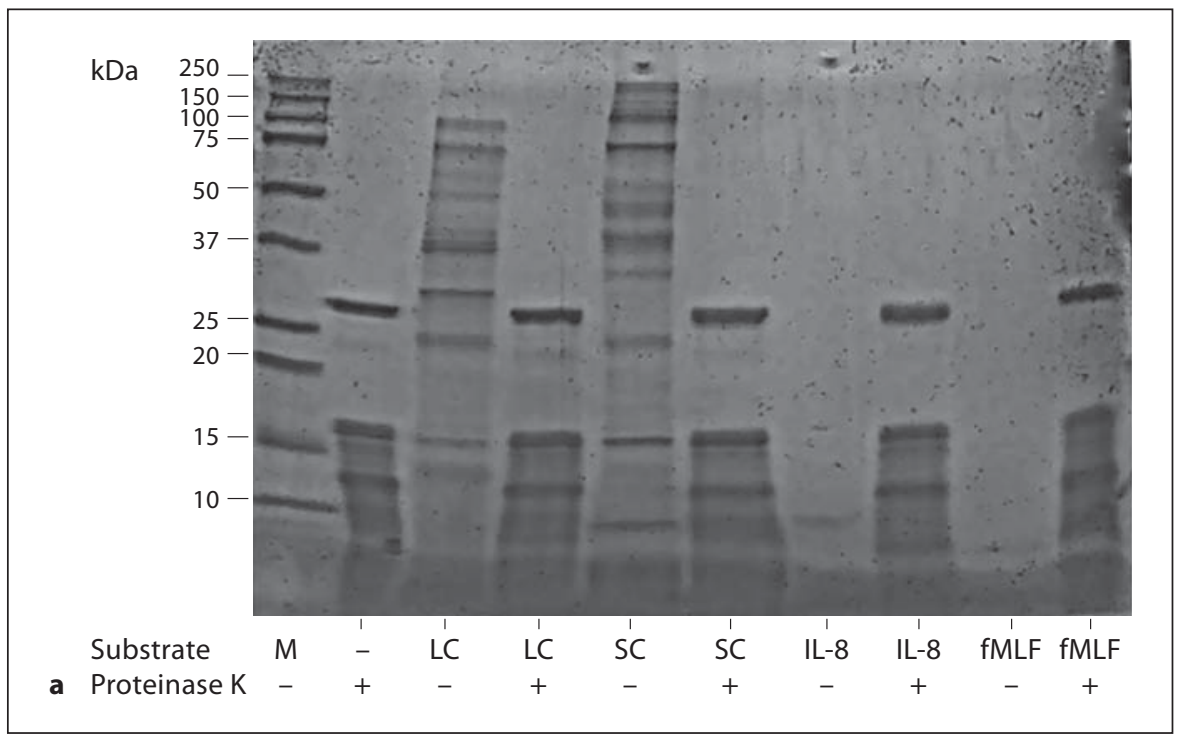

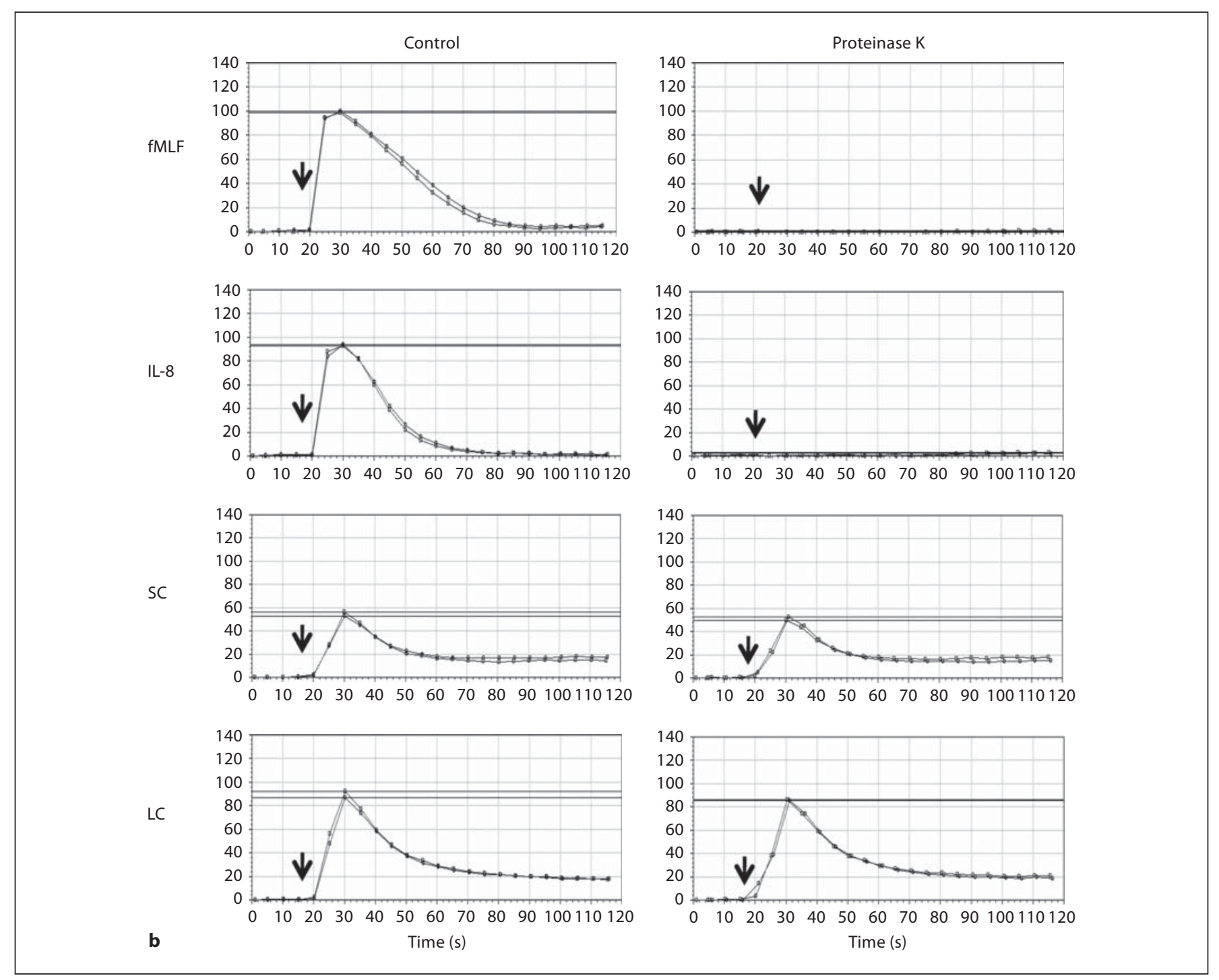


sites are rich in dendritic cells, macrophages and lymphocytes. In the context of gastrointestinal tract infections and inflammatory bowel disease, innate immune cells (neutrophils, macrophages, dendritic cells, and natural killer T cells) and adaptive immune cells (B cells and T cells) from peripheral blood are recruited to and activated in the mucosa, releasing proinflammatory cytokines [8]. Newly recruited leukocytes, particularly macrophages, express innate immune receptors and are highly responsive to microbial specific factors [23].

The gut microbiota may influence the mucosal immune system directly, for example by bacterial-leukocyte contact, or indirectly by release of leukocyte-activating factors such as N-formylpeptides, LPS and peptidoglycan, hypomethylated dinucleotides [5, 24], and shortchain fatty acids [13]. It may also influence the mucosal immune system by dendritic cell sampling of bacterial antigens $[25,26]$, or by interactions with epithelium which then are transduced to immune cells $[27,28]$. Our conclusion that the factors in the gut lumen responsible for leukocyte activation in our study are not solely Fpr agonists is based on the observation that responses were not affected when Fprl-deficient leukocytes were used as target cells, or when wild-type leukocytes were pretreated with pertussis toxin, which is known to inhibit Fprl and Fpr2 signaling. Further, the observation that the activity was resistant to proteinase $\mathrm{K}$ suggests that the responsible factors are not $\mathrm{N}$-formylpeptide agonists of Fprl, with the caveats that (1) the gel system used to assess protein degradation cannot resolve very small peptides, (2) some $\mathrm{N}$ formylpeptides are selective for Fpr2, which is also expressed on phagocytes [7, 17], and (3) proteinase K may produce active compounds that are distinct from those that were present before the digestion.
Additional studies with Fpr2 knockout mice and Fprl/2 double mutant mice, when they become available, as well as direct purification of the active factor(s), will be needed to definitively resolve the question of whether or not $\mathrm{N}$-formylpeptides in the gut lumen contribute to SC and LC activity. However, since proteinase $\mathrm{K}$ is a lowspecificity protease, and the factors are $<3.5 \mathrm{kDa}$ in size, it is reasonable to hypothesize that the active factor(s) are not proteins or peptides, and thus, not $\mathrm{N}$-formylpeptides.

In conclusion, we have found that the mouse gut lumen from both the small and large intestines contains small, potent, highly efficacious leukocyte chemotactic and activating factors that may be distinct for neutrophils and PBMCs, and that Fprl agonists, acetate and LPS may account for little if any of this activity. Such factors may be important for shaping leukocyte trafficking and function in the gut in both health and disease. Identifying such factors may provide new insights into the cellular and molecular mechanisms underlying gut homeostasis and new targets for drug development in infectious and inflammatory diseases of the gut.

\section{Acknowledgement}

This work was supported by funding from the Division of Intramural Research, National Institute of Allergy and Infectious Diseases, National Institutes of Health.

\section{Disclosure Statement}

T.O. is a recipient of a post-baccalaureate fellowship of the NIH Academy.

\section{References}

1 Hooper LV, Gordon JI: Commensal hostbacterial relationships in the gut. Science 2001;292:1115-1118.

2 Backhed F, Ding H, Wang T, Hooper LV, Koh GY, Nagy A, Semenkovich CF, Gordon JI: The gut microbiota as an environmental factor that regulates fat storage. Proc Natl Acad Sci USA 2004;101:15718-15723.

-3 Hooper LV, Wong MH, Thelin A, Hansson L, Falk PG, Gordon JI: Molecular analysis of commensal host-microbial relationships in the intestine. Science 2001;291:881-884.

4 Dziarski R: Recognition of bacterial peptidoglycan by the innate immune system. Cell Mol Life Sci 2003;60:1793-1804. $\checkmark 5$ Krieg AM, Yi AK, Matson S, Waldschmidt TJ, Bishop GA, Teasdale R, Koretzky GA, Klinman DM: CpG motifs in bacterial DNA trigger direct B-cell activation. Nature 1995; 374:546-549.

6 Schiffmann E, Corcoran BA, Wahl SM: Nformylmethionyl peptides as chemoattractants for leucocytes. Proc Natl Acad Sci USA 1975;72:1059-1062.

-7 Ye RD, Boulay F, Wang JM, Dahlgren C, Gerard C, Parmentier M, Serhan CN, Murphy PM: International union of basic and clinical pharmacology. 73. Nomenclature for the formyl peptide receptor (FPR) family. Pharmacol Rev 2009;61:119-161.
-8 Abraham C, Cho JH: Inflammatory bowel disease. N Engl J Med 2009;361:20662078.

9 Gao JL, Lee EJ, Murphy PM: Impaired antibacterial host defense in mice lacking the $\mathrm{N}$ formylpeptide receptor. J Exp Med 1999;189: 657-662.

10 Gao JL, Murphy PM: Species and subtype variants of the $\mathrm{N}$-formyl peptide chemotactic receptor reveal multiple important functional domains. J Biol Chem 1993;268: 25395-25401.

-11 Le Y, Murphy PM, Wang JM: Formyl-peptide receptors revisited. Trends Immunol 2002; 23:541-548. 
12 Hartt JK, Barish G, Murphy PM, Gao JL: Nformylpeptides induce two distinct concentration optima for mouse neutrophil chemotaxis by differential interaction with two $\mathrm{N}$-formylpeptide receptor (FPR) subtypes. Molecular characterization of FPR2, a second mouse neutrophil Fpr. J Exp Med 1999; 190:741-747.

13 Maslowski KM, Vieira AT, Ng A, Kranich J, Sierro F, Yu D, Schilter HC, Rolph MS, Mackay F, Artis D, Xavier RJ, Teixeira MM, Mackay CR: Regulation of inflammatory responses by gut microbiota and chemoattractant receptor GPR43. Nature 2009;461:12821286.

14 Rabiet MJ, Huet E, Boulay F: Human mitochondria-derived $\mathrm{N}$-formylated peptides are novel agonists equally active on FPR and FPRL1, while Listeria monocytogenes-derived peptides preferentially activate FPR. Eur J Immunol 2005;35:2486-2495.

15 Migeotte I, Communi D, Parmentier M: Formyl peptide receptors: a promiscuous subfamily of $G$ protein-coupled receptors controlling immune responses. Cytokine Growth Factor Rev 2006;17:501-519.

16 Babbin BA, Jesaitis AJ, Ivanov AI, Kelly D, Laukoetter M, Nava P, Parkos CA, Nusrat A: Formyl peptide receptor-1 activation enhances intestinal epithelial cell restitution through phosphatidylinositol 3-kinase-dependent activation of Rac1 and Cdc42. J Immunol 2007;179:8112-8121.
17 Southgate EL, He RL, Gao JL, Murphy PM, Nanamori M, Ye RD: Identification of formyl peptides from Listeria monocytogenes and Staphylococcus aureus as potent chemoattractants for mouse neutrophils. J Immunol 2008;181:1429-1437.

18 Mrsny RJ, Gewirtz AT, Siccardi D, Savidge T, Hurley BP, Madara JL, McCormick BA: Identification of hepoxilin A3 in inflammatory events: a required role in neutrophil migration across intestinal epithelia. Proc Natl Acad Sci USA 2004;101:7421-7426.

19 Mazmanian SK, Round JL, Kasper DL: A microbial symbiosis factor prevents intestinal inflammatory disease. Nature 2008;453: 620-625.

20 Tlaskalova-Hogenova H, Stepankova R, Hudcovic T, Tuckova L, Cukrowska B, Lodinova-Zadnikova R, Kozakova H, Rossmann P, Bartova J, Sokol D, Funda DP, Borovska D, Rehakova Z, Sinkora J, Hofman J, Drastich P, Kokesova A: Commensal bacteria (normal microflora), mucosal immunity and chronic inflammatory and autoimmune diseases. Immunol Lett 2004;93:97-108.

-21 Rimoldi M, Chieppa M, Salucci V, Avogadri F, Sonzogni A, Sampietro GM, Nespoli A, Viale G, Allavena P, Rescigno M: Intestinal immune homeostasis is regulated by the crosstalk between epithelial cells and dendritic cells. Nat Immunol 2005;6:507514.

22 McGuckin MA, Eri R, Simms LA, Florin TH, Radford-Smith G: Intestinal barrier dysfunction in inflammatory bowel diseases. Inflamm Bowel Dis 2009;15:100-113.
3 Smythies LE, Sellers M, Clements RH, Mosteller-Barnum M, Meng G, Benjamin WH, Orenstein JM, Smith PD: Human intestinal macrophages display profound inflammatory anergy despite avid phagocytic and bacteriocidal activity. J Clin Invest 2005;115:66-75.

24 Messina JP, Gilkeson GS, Pisetsky DS: Stimulation of in vitro murine lymphocyte proliferation by bacterial DNA. J Immunol 1991; 147:1759-1764.

25 Rescigno M, Rotta G, Valzasina B, RicciardiCastagnoli P: Dendritic cells shuttle microbes across gut epithelial monolayers. Immunobiology 2001;204:572-581.

-26 Rescigno M, Urbano M, Valzasina B, Francolini M, Rotta G, Bonasio R, Granucci F, Kraehenbuhl JP, Ricciardi-Castagnoli P: Dendritic cells express tight junction proteins and penetrate gut epithelial monolayers to sample bacteria. Nat Immunol 2001;2:361-367.

$27 \mathrm{He}$ B, Xu W, Santini PA, Polydorides AD, Chiu A, Estrella J, Shan M, Chadburn A, Villanacci V, Plebani A, Knowles DM, Rescigno M, Cerutti A: Intestinal bacteria trigger T cellindependent immunoglobulin $\mathrm{A}(2)$ class switching by inducing epithelial-cell secretion of the cytokine APRIL. Immunity 2007; 26:812-826.

28 McCormick BA, Colgan SP, Delp-Archer C, Miller SI, Madara JL: Salmonella typhimurium attachment to human intestinal epithelial monolayers: transcellular signalling to subepithelial neutrophils. J Cell Biol 1993; 123:895-907. 